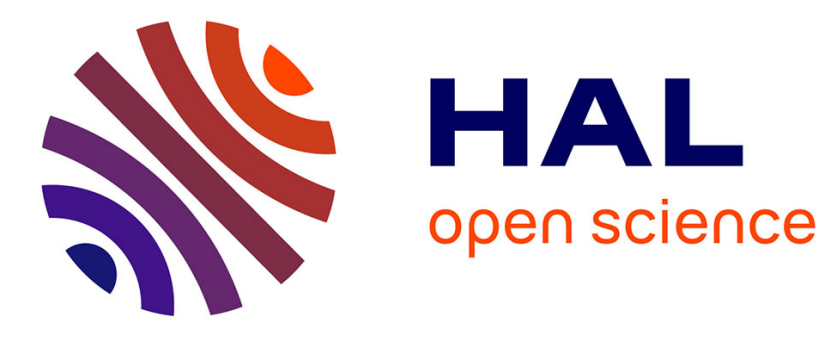

\title{
On dead-zone observers for linear plants
}

\author{
M. Cocetti, Sophie Tarbouriech, Luca Zaccarian
}

\section{To cite this version:}

M. Cocetti, Sophie Tarbouriech, Luca Zaccarian. On dead-zone observers for linear plants. American Control Conference (ACC 2018), Jun 2018, Milwaukee (WI), United States. pp.5138-5143, 10.23919/ACC.2018.8431895 . hal-01970889

\section{HAL Id: hal-01970889 \\ https://hal.laas.fr/hal-01970889}

Submitted on 6 Jan 2019

HAL is a multi-disciplinary open access archive for the deposit and dissemination of scientific research documents, whether they are published or not. The documents may come from teaching and research institutions in France or abroad, or from public or private research centers.
L'archive ouverte pluridisciplinaire HAL, est destinée au dépôt et à la diffusion de documents scientifiques de niveau recherche, publiés ou non, émanant des établissements d'enseignement et de recherche français ou étrangers, des laboratoires publics ou privés. 


\title{
On dead-zone observers for linear plants
}

\author{
M. Cocetti, S. Tarbouriech, L. Zaccarian
}

\begin{abstract}
We propose a new class of Luenberger-like observers for LTI plants which are robust with respect to measurements affected by high-frequency noise. These observers have the classical Luenberger structure but the output injection term is corrected by means of an adaptive dead-zone. The dead-zone "cuts" part of the noise using the dead-band. The dead-zone levels are dynamically adapted according to noise, establishing a trade-off between speed of convergence and sensitivity to noise. We show that both the observer gain and the adaptation parameters can be obtained solving a Linear Matrix Inequality (LMI), whose feasibility only requires detectability of the plant. The parameters obtained through this optimization procedure ensure Uniform Global Asymptotic Stability of the origin in the error coordinates. The effectiveness of the approach is shown by means of a numerical example.
\end{abstract}

\section{INTRODUCTION}

Robustly reconstructing the state of a plant from input-output measurements is one of the most fundamental problems in control theory. State observers have been developed to address this problem and more than 40 years of research produced a large number of outstanding results. The first milestone has been obtained by David Luenberger in [1], where the problem of state estimation for Single Input Single Output (SISO) linear plant has been solved by mean of a dynamic filter, today called Luenberger observer. A few years later the observer has been extended to the multi-input multi-output case in [2] and researchers immediately recognized the importance of these results and the fundamental connection with the early introduced concept of observability. Soon it became clear that observers could play a fundamental role in output feedback stabilization and people started combining observers with state feedback design obtaining dynamic output stabilizers.

This idea of replacing the state of the plant with an estimate provided by the observer had a tremendous impact in the following years, and rapidly become a standard design procedure. However a big open question at that time was the robustness of this approach. Indeed, even if the state feedback and the observer are designed to possess a large stability margin, their interconnection may be extremely fragile [3]. Trying to solve this problem Doyle and Stein developed the loop transfer recovery approach [4] and high-gain observers were born. High-gain observers overcome the problem providing an intrinsic robustness with respect to modeling errors, moreover they recover the performance achievable by state feedback if a sufficiently large gain is selected.

These remarkable properties motivated a lot of work aiming to extending high-gain observers to the nonlinear setting. First works in this direction appeared at the beginning of the ' 90 in [5], [6], and in the subsequent years high-gain observers became popular tools to solve nonlinear control problems ranging from stabilization to tracking and regulation. This great impact was also possible thanks to output stabilization techniques developed in [7] and to the semiglobal separation principle obtained in [8], [9]. These results allow to design feedback laws as if the state were available and then use an estimate provided by a sufficiently fast observer. The prices to pay for this flexibility are essentially twofold: first, peaking phenomena, and second high sensitivity to measurements noise. The latter was already well known in the framework of linear observers [10] and entails the classical trade-off between bandwidth and noise rejection. Indeed, as the observer gain increases, the convergence rate increases as well but the observer's behavior approximates a differentiator and enormously amplifies the noise.

Still, high-gain observers are Input to State Stable (ISS) with respect to measurement noise. The ISS property guarantees that trajectories remain bounded as long as the disturbances remain bounded as well. These bounds have been recently investigated in [11], [12], where the authors propose to study the effect of measurement noise on the estimation error and in a feedback control framework.

High sensitivity to noise is one of the main limitations for applicability of high-gain techniques in real scenarios. To overcome this sensibility many solutions have been proposed and they can be classified in three groups.

1) Multi-observer solution,

2) Switched gain solution,

3) Adaptive gain solution,

4) Nonlinear or time-varying output injection.

In the multi-observer solution a bank of different observers (a multi-observer) is implemented. Each observer has different sensitivity to noise and convergence speed and a supervisor (which monitors a pre-designed selection criterion) chooses

M. Cocetti, and L. Zaccarian are with the Department of Industrial Engineering, 9 Sommarive, University of Trento, Trento, IT, 38123. Email: (matteo.cocetti, luca.zaccarian) dunitn.com and also with LAAS-CNRS, University of Toulouse, CNRS, Toulouse, France. S. Tarbouriech is with LAAS-CNRS, University of Toulouse, CNRS Toulouse, France. Email: tarbour@laas.fr

This research was supported by grant PowerLyap funded by CaRiTRo and DRTS Transmission Systems. 
which observer is providing the best estimate at any given time. This approach has been used in [13] and recently with the goal of obtaining robustness with respect to an uncertain plant in [14] (each observer is designed for a different value of the uncertain parameter).

In the switching approach the observer gain is dynamically selected among two or more pre-designed values. For example it is possible to use a large observer gain for transient estimation and a small one for steady-state performance [15]. However, there are many design challenges in using this approach, e.g., it is hard to properly select the switching time/mechanism (like trigger or threshold based mechanism) and the intrinsic discontinuous behavior of the observer is dangerous when combined with phenomena as peaking. A similar, but simpler, approach has been also proposed in [16] where a piecewise linear gain function is used.

The limiting case of arbitrarily fast switching among different observers or different observer gains can be though as a continuous adaptation. This approach has been used for example in [17], [18], where the observer gain is dynamically adapted according to the difference $(\hat{y}-y)$. Usually these schemes behave better than classical high-gain observers, but the performance heavily depends on the chosen adaptation law.

Finally non-linear, homogeneous and adaptive output injection/correction terms have been considered in [19], [20].

Similarly, in this work we propose an approach that does not change the observer gain but employs an adaptive non-linear output injection term. For the proposed observer the term $(\hat{y}-y)$ passes through an artificial dead-zone function, whose dead-band is dynamically adapted. The benefit of the proposed solution is that high-frequency noise remains partially (or completely) trapped inside the dead-band and has a mitigated (or completely no) effect on the observer dynamics. Moreover, due to the adaptation mechanism, the dead-band amplitude is an indirect measure of the noise level and provides useful information that can be used for higher levels tasks.

The dead-zone mechanism has a destabilizing effect, since in a ball around the zero estimation error the observer essentially runs in an open-loop fashion. However, if the parameters are carefully tuned, and the adaptation is fast enough, the dead-zone rapidly converges to the identity function and we retrieve the classical Luenberger observer in a noise free scenario.

The use of dead-zones is strongly motivated by a large amount of results developed for linear systems subject to sector bounded nonlinearities [21], e.g., global and sector conditions [22], [23], [24]. These tools have been extensively used in combination with Linear Matrix Inequalities (LMIs), [25] in the context of anti-windup design, but rarely employed for observers, even if there are few remarkable exceptions [26], [27].

For example in [26] the authors propose a two observers architecture that is robust with respect to quantization noise. The interplay among the two observers is ruled by a constant dead-zone whose amplitude is a-priori fixed according to the quantization step. In [27] a novel Luenberger-like Stubborn Observer (SO) is proposed. Stubborn Observers has an output injection term that is artificially saturated with an adaptive threshold. The goal of this mechanism is to reduce sensitivity to outliers, however this solution has a little effect on low amplitude high-frequency noise (due to linearity of the saturation around the origin).

Compared to [27] in this work we pursue a complementary goal and we introduce an adaptive dead-zone output injection term. The resulting observer named Dead-zone Observer (DO) has the remarkable feature of rejecting high-frequency noise. We show that the synthesis of this class of observers is always possible for detectable LTI plants and it can be formulated as feasibility of a proper LMI.

The paper is organized as follows. Section II presents the problem formulation. Section III contains the main results of the paper and the related proofs. Section IV provides some guidelines for the observer synthesis optimization. An example is provided in Section V. Finally, conclusions are offered in Section VI.

Notation: Let $\mathbb{R}^{n}$ denote the set of real vectors of dimension $n$. Given a constant $c \in \mathbb{R}$ we write $\mathbb{R}_{\geq c}$ to denote the subset $[c, \infty) \subset \mathbb{R}$. Given two vectors $x \in \mathbb{R}^{n}, y \in \mathbb{R}^{m},(x, y):=\left[x^{\top} y^{\top}\right]^{\top} \in \mathbb{R}^{n+m}$. For a matrix $M \in \mathbb{R}^{n \times m}, M^{\top}$ denotes its transpose. For square invertible matrices $M \in \mathbb{R}^{n \times n}, M^{-1}$ denotes the inverse of $M$ and $M^{-\top}$ its inverse transpose, $M>0$ $(M \geq 0)$ denotes positive definiteness (semi-definiteness) of $M$, and $\operatorname{He}(M):=\left(M+M^{\top}\right)$. We denote by Diag ${ }_{>0}^{n}$ (resp. $\operatorname{Diag}_{\geq 0}^{n}$ ) the set of diagonal positive (resp. semi) definite matrices of dimension $n \times n$, and by $\operatorname{Sym}_{>0}^{n}\left(\mathrm{resp} . \mathrm{Sym}_{\geq 0}^{n}\right) \mathrm{the}^{n}$ set of symmetric positive (resp. semi) definite matrices of dimension $n \times n$. The vectors $\mathbf{1}, \mathbf{2}$ are defined respectively as $\mathbf{1}:=(1,1, \ldots, 1)^{\top} \in \mathbb{R}^{p}$ and $\mathbf{2}:=2 \mathbf{1} \in \mathbb{R}^{p}$. Finally $\lambda_{k}(M)$ denotes the $k$-th eigenvalue of matrix $M$.

\section{PROBLEM FORMULATION}

In this paper we consider a continuous time Linear Time Invariant (LTI) system ("plant") of the following form,

$$
\left\{\begin{array}{l}
\dot{x}=A x+B u \\
y=C x+D u,
\end{array}\right.
$$

where $x \in \mathbb{R}^{n}$ is the state, $u \in \mathbb{R}^{m}$ is the input and $y \in \mathbb{R}^{p}$ is the measured output. In [27] the authors proposed a new type of Luenberger-like observer named Stubborn Observer (SO). In the stubborn architecture the output injection term is artificially saturated to reduce the effect of outliers in the error estimation dynamics and saturation levels are dynamically adapted to obtain Global Asymptotic Stability (GAS). It is worth to notice that the adaptation is necessary to obtain global 
results, see [28], [27]. Compared to [27] here we propose a complementary Luenberger-like observer where the output injection is "dead-zonated" and the dead-zone levels can be designed in a more independent way. The resulting observer is effective in dealing with high-frequency noise at the plant output. The Dead-zone Observer (DO) that we propose has the following form,

$$
\left\{\begin{array}{l}
\dot{\hat{x}}=A \hat{x}+B u+L \mathrm{dz}_{\sqrt{\sigma}}(\hat{y}-y) \\
\hat{y}=C \hat{x}+D u
\end{array}\right.
$$

where $\hat{x} \in \mathbb{R}^{n}$ is the estimated state, $\hat{y} \in \mathbb{R}^{p}$ is the estimated output and $\sigma \in \mathbb{R}_{>0}^{p}$ is a vector, whose entries are non-negative and define the amplitude of the dead-zone. Matrix $L \in \mathbb{R}^{n \times p}$ is the classical observer gain. The function $\mathrm{dz} \sqrt{\sigma}: \mathbb{R}^{p} \rightarrow \mathbb{R}^{p}$ is a decentralized vector-valued dead-zone defined as follows

$$
\mathrm{dz}_{\sqrt{\sigma}}(y):=\left[\begin{array}{c}
\mathrm{dz}_{\sqrt{\sigma_{1}}}\left(y_{1}\right) \\
\vdots \\
\mathrm{dz}_{\sqrt{\sigma_{p}}}\left(y_{p}\right)
\end{array}\right],
$$

and where $\sqrt{\sigma}:=\left(\sqrt{\sigma_{1}}, \ldots, \sqrt{\sigma_{p}}\right) \in \mathbb{R}^{p}$ is a component-wise square root. For $\sigma$ we propose the following adaptation law

$$
\dot{\sigma}=-\Lambda \sigma+\mathbf{1}(\hat{y}-y)^{\top} R(\hat{y}-y), \quad \sigma \in \mathbb{R}_{\geq 0}^{p}
$$

where $\Lambda \in \operatorname{Diag}_{>0}^{p}$ is a diagonal positive definite matrix, $R \in \operatorname{Sym}_{\geq 0}^{p}$ is a positive semi-definite matrix. The constraint $\sigma \in \mathbb{R}_{\geq 0}^{p}$ means that $\sigma$ belongs to the closed $p$-dimensional positive orthant, which is an invariant set for (3). It is worth to notice that non-negativity of $\sigma$ makes the square root $\sqrt{\sigma}$ always well defined.

The idea behind the observer in (2) is quite intuitive and entails the fact that the dead-zone provides a zero output correction term around $(\hat{y}-y)=0$. This dead-zone mechanism has a strong filtering action and a destabilizing effect on the observer dynamics. For this reason a fast enough $\sigma$-adaptation in (3) is necessary to ensure convergence in the "small", otherwise for a fixed (o slowly converging) dead-zone, signals $\hat{y}$ and $y$ which are close enough would never synchronize. It is worth to notice that the adaptive mechanism (3) is designed to weigh two antagonistic effects: first, "adaptation speed" selected by $\Lambda$, and second, "filtering action" tuned by $R$. Intuitively selecting $\Lambda$ large enough, and $R$ sufficiently small we can recover a classical Luenberger observer. On the other hand, setting $\Lambda$ small and $R$ large we slow down the convergence rate but we greatly increase the filtering capability.

\section{MAIN RESUlTS}

In this section we prove a few good properties for the DO in (2). In particular we show that with a detectable plant $\Lambda$ and $R$ can always be chosen in order to obtain GAS of the error dynamics.

\section{A. Global Asymptotic Stability (GAS)}

Given the DO (2) and the adaptation law (3) we cast the design of the adaptation law (3) as a Linear Matrix Inequality (LMI). We show that this tuning ensures Global Asymptotic Stability (GAS) of the observer error dynamics and of the adaptive parameter $\sigma$. Towards this goal let us define the estimation error as $e=\hat{x}-x$ and recall that

$$
\operatorname{sat}_{\sqrt{\sigma}}(y)+\mathrm{dz}_{\sqrt{\sigma}}(y)=y,
$$

then, after few manipulations, we obtain the following equivalent representation for (2) interconnected with (3),

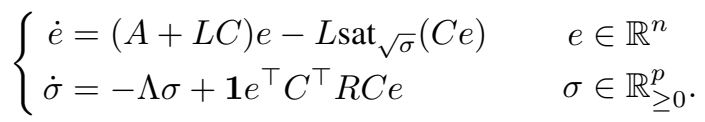

Notice that (4) represents the error dynamics of a classical Luenberger observer plus a perturbation term whose amplitude is ruled by $\sqrt{\sigma}$. The goal of the following formulation is to simultaneously design parameters $\Lambda, R$ and $L$ such that (4) is GAS to the origin. What follows is the main result of the paper.

Theorem 1. Consider the following LMI in the optimization variables $P \in \mathrm{Sym}_{>0}^{n}, X \in \mathbb{R}^{n \times p}, \Lambda \in \mathrm{Diag}_{>0}^{p}, R \in \mathrm{Sym}_{\geq 0}^{p}$, $U \in \operatorname{Diag}_{\geq 0}^{p}$,

$$
\operatorname{He}\left[\begin{array}{cc}
P A+X C+\mathbf{1}^{\top} \mathbf{1} C^{\top} R C & -X \\
U C & -U-\Lambda
\end{array}\right]<0,
$$

then any feasible solution to (5), together with the choice

$$
L:=P^{-1} X
$$

provides a design selection that makes (4) globally asymptotically stable to the origin. 
Proof. As a first step we notice that strict negativity of (5) implies that there exist a sufficiently small $c_{0} \in \mathbb{R}_{>0}$ such that,

$$
\mathrm{He}\left[\begin{array}{cc}
P A+X C+\mathbf{1}^{\top} \mathbf{1} C^{\top} R C & -X \\
U C & -U-\left(1-c_{0}\right) \Lambda
\end{array}\right] \leq-2 c_{0} I
$$

Then, let us consider the candidate Lyapunov function $V(e, \sigma):=e^{\top} P e+\mathbf{2}^{\top} \sigma$, which satisfies the following bounds,

$$
\alpha_{1}\|(e, \sqrt{\sigma})\|^{2} \leq\left[\begin{array}{c}
e \\
\sqrt{\sigma}
\end{array}\right]^{\top}\left[\begin{array}{cc}
P & 0 \\
0 & 2 I
\end{array}\right]\left[\begin{array}{c}
e \\
\sqrt{\sigma}
\end{array}\right] \leq \alpha_{2}\|(e, \sqrt{\sigma})\|^{2},
$$

where $\alpha_{1}:=\min \left\{\lambda_{\min }(P), 2\right\}$, and $\alpha_{2}:=\max \left\{\lambda_{\max }(P), 2\right\}$. The Lie derivative along trajectories yields

$$
\begin{aligned}
\dot{V}(e, \sigma)= & e^{\top}(A+L C)^{\top} P e+e^{\top} P(A+L C) e \\
& -2 e^{\top} P L \text { sat }_{\sqrt{\sigma}}(C e) \\
& -\mathbf{2}^{\top} \Lambda \sigma+\mathbf{2}^{\top} \mathbf{1} e^{\top} C^{\top} R C e .
\end{aligned}
$$

To enforce (strict) negativity of (8) we introduce some conservativeness. First, we consider a global sector condition for cone bounded nonlinearities, [22], and second we use the simple observation that the saturation levels are proportional to $\sqrt{\sigma}$ and thus $\operatorname{sat}_{\sqrt{\sigma}}^{\top}(\mathrm{Ce})$ sat $_{\sqrt{\sigma}}(\mathrm{Ce})$ never exceed $\mathbf{1}^{\top} \sigma$. These observations translate into the following inequalities

$$
\begin{aligned}
\operatorname{sat}_{\sqrt{\sigma}}^{\top}(C e) U\left(C e-\operatorname{sat}_{\sqrt{\sigma}}(C e)\right) & \geq 0, \\
\mathbf{1}^{\top} \Lambda \sigma-\operatorname{sat}_{\sqrt{\sigma}}^{\top}(C e) \Lambda \operatorname{sat}_{\sqrt{\sigma}}(C e) & \geq 0,
\end{aligned}
$$

where we used $U \in \operatorname{Diag}_{>0}^{p}, \Lambda \in \operatorname{Diag}_{>0}^{p}$. Multiplying (9a) by two and (9b) by $2\left(1-c_{0}\right)$, and summing up to (8) we obtain the following upper-bound, which uses $X:=P L \in \mathbb{R}^{n \times p}$,

$$
\begin{aligned}
\dot{V}(e, \sigma) \leq & {\left[\begin{array}{c}
e \\
\operatorname{sat}_{\sqrt{\sigma}}(C e)
\end{array}\right]^{\top}\left[\begin{array}{c}
\operatorname{He}(P(A+L C))+\mathbf{2}^{\top} \mathbf{1} C^{\top} R C \\
-L^{\top} P+U C \\
-P L+C^{\top} U \\
\\
-2 U-2\left(1-c_{0}\right) \Lambda
\end{array}\right]\left[\begin{array}{c}
e \\
\operatorname{sat}_{\sqrt{\sigma}}(C e)
\end{array}\right]-2 c_{0} \mathbf{1}^{\top} \Lambda \sigma } \\
\leq & -2 c_{0}\left(\|e\|^{2}+\left\|\operatorname{sat}_{\sqrt{\sigma}}(C e)\right\|^{2}+\mathbf{1}^{\top} \Lambda \sigma\right) \\
\leq & -2 c_{1}\left(\|e\|^{2}+\|\sqrt{\sigma}\|^{2}\right)=-2 c_{1}\|(e, \sqrt{\sigma})\|^{2}
\end{aligned}
$$

where we used (7), and we selected $c_{1} \in \mathbb{R}_{>0}$ sufficiently small.

\section{B. Feasibility}

Theorem 1 provides only a sufficient condition to enforce Global Asymptotic Stability, however it turns out that feasibility of (5) can be exactly characterized.

Proposition 1. Inequality (5) is feasible if and only if pair $(C, A)$ is detectable.

Proof. Necessity follows from standard detectability results. Indeed, if pair $(C, A)$ is not detectable, no asymptotic observer exists for the plant state and therefore condition (5) is infeasible. To prove sufficiency we first use [29, Thm 16.6] to get that detectability of $(C, A)$ implies the existence of a matrix $P \in \mathrm{Sym}_{>0}^{n}$ satisfying

$$
A^{\top} P+P A-C^{\top} C<0 \text {. }
$$

From (5) considering the selection $X=-C^{\top} / 2, U=0, R=0$, and $\Lambda=\lambda I / 4$, where $\lambda \in \mathbb{R}_{>0}$, we obtain the following

$$
\left[\begin{array}{cc}
A^{\top} P+P A-C^{\top} C & C^{\top} / 2 \\
C / 2 & -\lambda I / 4
\end{array}\right]<0 .
$$

Using a Shur complement, we obtain $-\lambda I / 4<0$ and

$$
A^{\top} P+P A+\left(\lambda^{-1}-1\right) C^{\top} C<0
$$

which is feasible for $\lambda$ sufficiently large due to (10).

Remark 1. It is worth to notice that, under the detectability assumption, condition (5) is always feasible even when a stabilizing gain $L$ is a priori fixed. In this case we can think of (2) as a dead-zone augmentation, i.e., an adaptive output injection mechanism able to augment the performance of a pre-designed observer. The LMI in (5) can be still used as a design tool fixing a stabilizing $L$ (i.e., such that $(A+L C)$ is Hurwitz) and substituting $X=P L$. The arising LMI is feasible and provides a convenient way to design the adaptation parameters $\Lambda$ and $R$. 


\section{Input to State Stability in the presence of measurement noise.}

In this subsection we introduce the presence of an additive noise $v \in \mathbb{R}^{p}$ affecting the plant output $y$, and we study the effect on the dead-zone observer proposed in (2). In particular we show that (4) is Input to State Stable (ISS) from $v$ to $(e, \sigma)$. For this purpose let us consider an additive disturbance $v$, with the modified output equation $y=C x+D u+v$. Then, following the same steps as in Subsection III-A, we obtain the following representation for the closed-loop in the error coordinates,

$$
\left\{\begin{array}{l}
\dot{e}=(A+L C) e-L\left(v+\operatorname{sat}_{\sqrt{\sigma}}(C e-v)\right) \\
\dot{\sigma}=-\Lambda \sigma+\mathbf{1}(C e-v)^{\top} R(C e-v), \quad \sigma \in \mathbb{R}_{\geq 0}^{p} .
\end{array}\right.
$$

Equation 11 shows that the disturbance has a quadratic effect on the $\sigma$ dynamics and has a reduced effect on the $e$ dynamics due to $\operatorname{sat}_{\sqrt{\sigma}}(C e-v)$.

Proposition 2. System (11) with, $L, \Lambda$, and $R$ designed according to (5), is Input to State Stable (ISS) from $v$ to (e, $\sigma)$.

Proof. Consider the same Lyapunov function as in the proof of Theorem 1, then the Lie derivative along the flow in presence of $v$ yields,

$$
\begin{aligned}
\dot{V}(e, \sigma) \leq & -2 c_{1}\left(\|e\|^{2}+\|\sqrt{\sigma}\|^{2}\right)+\mathbf{2}^{\top} \mathbf{1} v^{\top} R v-2 e^{\top} P L v \\
& -\mathbf{2}^{\top} \mathbf{2} e^{\top} C^{\top} R v-2 \operatorname{sat}_{\sqrt{\sigma}}^{\top}(C e-v) U v \\
\leq & -2 c_{1}\left(\|e\|^{2}+\|\sqrt{\sigma}\|^{2}\right)+c_{2}\|v\|^{2}+c_{3}\|e\|\|v\| \\
& +c_{4}\|\sqrt{\sigma}\|\|v\|,
\end{aligned}
$$

where we selected $c_{2}:=\left\|\mathbf{2}^{\top} \mathbf{1} R\right\|, c_{3}:=\|2 P L\|+\left\|\mathbf{2}^{\top} \mathbf{2} C R\right\|, c_{4}:=\|2 U\|$. Using the following Young's inequalities

$$
\begin{aligned}
\|e\|\|v\| & \leq \frac{c_{1}}{2 c_{3}}\|e\|^{2}+\frac{c_{3}}{2 c_{1}}\|v\|^{2} \\
\|v\|\|\sqrt{\sigma}\| & \leq \frac{c_{1}}{2 c_{4}}\|\sqrt{\sigma}\|^{2}+\frac{c_{4}}{2 c_{1}}\|v\|^{2},
\end{aligned}
$$

we upper-bound (12) as follows

$$
\begin{aligned}
\dot{V}(e, \sigma) \leq & -c_{1}\left(\|e\|^{2}+\|\sqrt{\sigma}\|^{2}\right)-\frac{c_{1}}{2}\left(\|e\|^{2}+\|\sqrt{\sigma}\|^{2}\right) \\
& +\left(2 c_{1}\right)^{-1}\left(2 c_{1} c_{2}+c_{4}^{2}+c_{3}^{2}\right)\|v\|^{2} \\
\leq & -c_{1}\left(\|e\|^{2}+\|\sqrt{\sigma}\|^{2}\right) \leq 0
\end{aligned}
$$

which holds for all $\|(e, \sqrt{\sigma})\|^{2} \geq\left(c_{1}\right)^{-2}\left(2 c_{1} c_{2}+c_{4}^{2}+c_{3}^{2}\right)\|v\|^{2}=c_{5}^{2}\|v\|^{2}$. We conclude that (11) is ISS from $v$ to $(e, \sqrt{\sigma})$ with an ISS gain proportional to $c_{5}$.

Roughly speaking Proposition 2 guarantees that for any bounded disturbance, the trajectories of (2) remain bounded. This is a fundamental property, especially for non-linear observers where instability may occur due to arbitrarily small measurement noise, see for example [30]. We also notice that the ISS property ensures that the performance degradation is gradual, which is another remarkable property of (11).

\section{LMI-BASED SYNTHESIS.}

In this section we provide some tuning guidelines for the Dead-zone Observer proposed in (2). Toward this goals let us define $M \in \mathbb{R}^{n \times n}$ as follows,

$$
M:=P A+X C \text {. }
$$

As a first step, we suggest to select a proper interval for the desired convergence rate. This can be easily done choosing the parameters $\alpha_{\min }, \alpha_{\max } \in \mathbb{R}_{>0}, \alpha_{\max }>\alpha_{\min }$, and considering the following inequalities

$$
\begin{aligned}
& \operatorname{He}(M)+2 \alpha_{\min } P \leq 0 \\
& \operatorname{He}(M)+2 \alpha_{\max } P \geq 0 .
\end{aligned}
$$

From the standard pole placement LMI formulation, see [31], it follows that $-\alpha_{\max } \leq \operatorname{Re}\left(\lambda_{k}(A+L C)\right) \leq-\alpha_{\text {min }}$, for $k=1, \ldots, n$. Moreover, to prevent the closed-loop poles from having a too large imaginary component, we suggest to consider the additional constrain

$$
\left[\begin{array}{ll}
\left(M+M^{\top}\right) \sin \theta & \left(M-M^{\top}\right) \cos \theta \\
\left(M^{\top}-M\right) \cos \theta & \left(M+M^{\top}\right) \sin \theta
\end{array}\right] \leq 0
$$

parametrized by $\theta \in(0, \pi / 2)$. Equation (14) ensures that the closed-loop damping factor $\xi \in \mathbb{R}$ associated to $(A+L C)$ satisfies $\xi \geq \cos (\theta)$, see [31]. 
Remark 2. Notice that (14) and (13) are not too restrictive for the feasibility of (5). Indeed if we assume observability of the pair $(C, A)$ the closed-loop poles of $(A+L C)$ can be arbitrarily placed and (14), (13) are automatically feasible.

We also impose an upper-bound on the adaptation speed fixing the parameter $\lambda_{\max } \in \mathbb{R}_{>0}$. This constraint avoids an excessive time scale separation among the plant and the adaptation mechanism. A trivial selection is to take $\lambda_{\text {max }}$ as a multiple of $\alpha_{\max }$. The corresponding inequality is the following,

$$
0<\Lambda \leq \lambda_{\max } I
$$

Finally we consider the standard positive definiteness requirement for $P$ and positive semi-definiteness for $R$ and $U$ as follows

$$
0<P=P^{\top}, \quad 0 \leq R=R^{\top}, \quad 0 \leq U=U^{\top}
$$

We propose to design the Dead-zone Observer according to the following heuristic optimization problem,

$$
\begin{aligned}
& \sup _{P, X, \Lambda, R} \operatorname{tr}(R) \\
& \text { subject to: }
\end{aligned}
$$

$$
(5),(13),(14),(15),(16) \text {. }
$$

The intuitive idea is to maximize the effect of disturbances on the $\sigma$ dynamics, so that the largest possible fraction of $v$ is trapped in the dead-band reducing the noise effect on the estimation error dynamics.

\section{AN EXAMPLE}

In this section we compare a classical Luenberger observer with the proposed Dead-zone Observer. We consider the following marginally stable plant

$$
\left[\begin{array}{l|l}
A & B \\
\hline C & D
\end{array}\right]=\left[\begin{array}{cc|c}
0 & 1 & 0 \\
-1 & 0 & 1 \\
\hline 1 & 1 & 0
\end{array}\right]
$$

The Luenberger observer gain $L$ has been designed using a standard Riccati equation where the weights have been selected to put more emphasis on a fast response $(100 I$ and $I)$. The resulting observer gain is $L=[3.89-13.59]^{\top}$. According to Remark 1, the same $L$ has been used for both the Luenberger and the Dead-zone Observer. Please notice that since $L$ is already fixed, the optimization problem in (17) does not involve $X$, which is therefore replaced by $P L$, see Remark 1 . The maximum adaptation speed has been fixed to $\lambda_{\max }=100$ and $\alpha_{\min }=0.9538=-0.95 \lambda_{\max }(A+L C)$. The constraint $(13 \mathrm{~b})$ has been disregarded since the closed-loop poles are already fixed by $L$. Solving (17) for $\Lambda$ and $R$ we obtain the following values $\Lambda=99.98$ and $R=84.16$.

For our simulation we consider the plant (1) forced by a sinusoidal input $u(t)=\sin (2 \pi t)$, with a randomly generated initial condition equal to $x(0)=[3.532 .86]^{\top}$. The initial conditions $\hat{x}(0)$ and $\sigma(0)$ have been selected to be zero. We corrupted the output measurements $y$ by noise generated by the Simulink block Uniform Random Number with sampling time and output range equal to $t_{\mathrm{s}}=0.0001 \mathrm{~s}$, and \pm 100 . In simulation the noise starts at time $t_{\text {start }}=12$ and ends at $t_{\text {end }}=20$.

A comparison among the true and estimated state for the two observers is shown in Figure 1. Figure 2 shows the estimation error where the benefit of the dead-zone mechanism can be better appreciated. Finally, Figure 3 reports the adaptation of $\sqrt{\sigma}$.

To compare the two observers we propose a tracking Error to Noise Ratio (ENR) which is inspired by the classical concept of Signal to Noise Ratio (SNR). The ENR is formally defined as follows

$$
\mathrm{ENR}:=\frac{\mathbb{E}\left\{e^{\top} e\right\}}{\mathbb{E}\left\{v^{\top} v\right\}}
$$

where $\mathbb{E}\{\cdot\}$ denotes the expectation operator. The value of the expectations has been obtained simply computing the average of the signals over the interval $\left[t_{\text {start }}, t_{\text {end }}\right]$. The ENRs for the two observers are reported in the table below. We can notice

\begin{tabular}{llr}
\hline & Luenberger & Dead-zone \\
\hline ENR & $3.65 \times 10^{-4}$ & $8.53 \times 10^{-5}$ \\
\hline
\end{tabular}

that the dead-zone mechanism successfully improves the tracking error to noise ratio. 

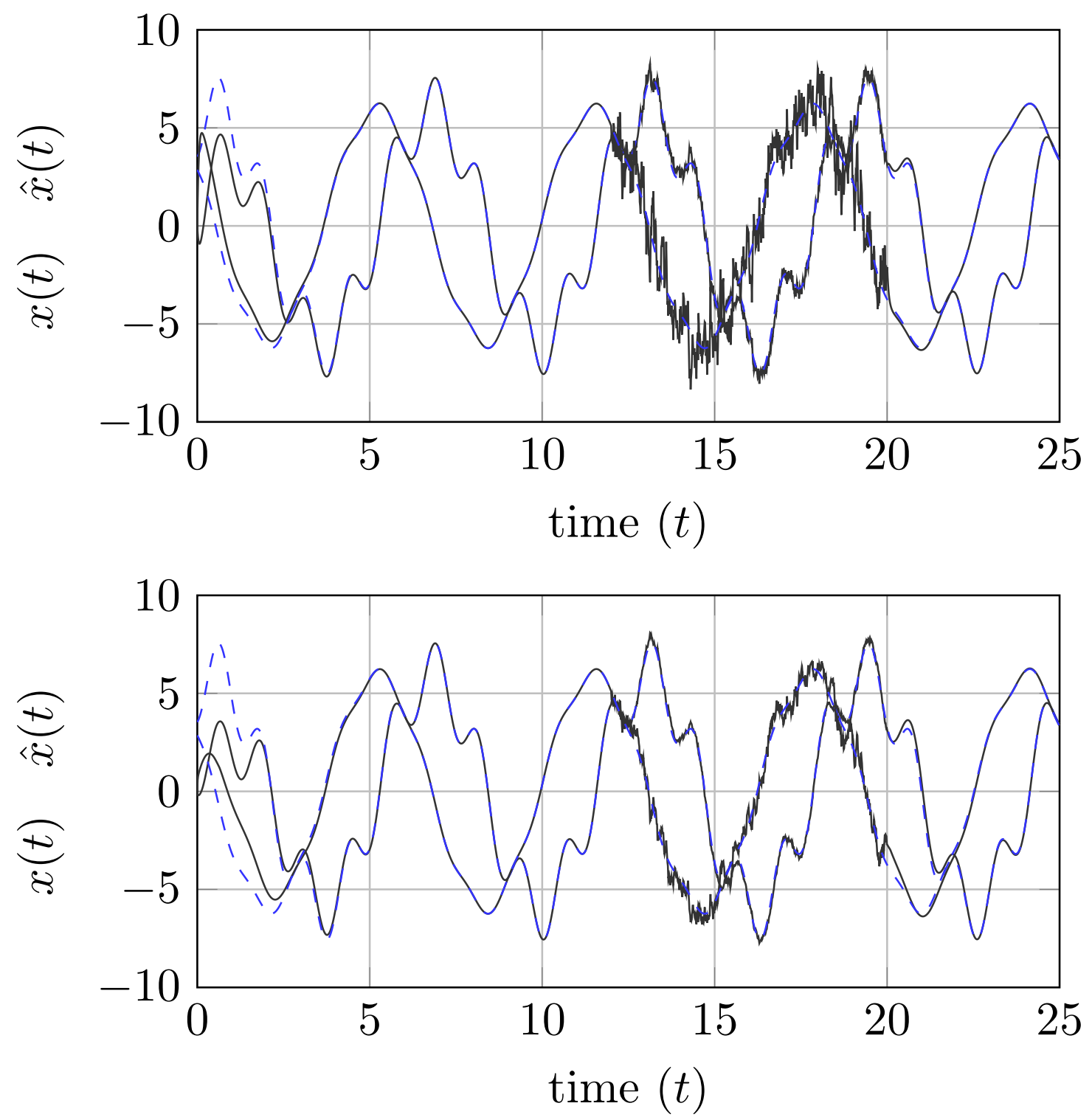

Fig. 1. Estimated (black) and true (blue) state of Luenberger observer (above) and Dead-zone Observer (below). Notice the significant reduction in the estimation error due to the dead-zone adaptation.

\section{CONCLUSION}

In this paper we presented a new class of adaptive observers for LTI plants. The main idea is to use an adaptive dead-zone function applied to the output injection term. This approach is in a sense complementary to the mechanism proposed in [27], where a saturation function has been used in the same setting. The proposed Dead-zone Observer is especially suitable to reduce sensitivity to high-frequency noise without sacrificing too much the convergence speed. We proved that Dead-zone Observers are not restrictive for LTI plants and can always be designed under standard (and necessary) detectability assumption. We also provide a heuristic LMI-based synthesis for the simultaneous design of the observer gain plus the adaptation parameters. An academic example shows the effectiveness of the proposed solution. Future research will involve extension of the proposed methodology to uniformly observable non-linear systems.

\section{REFERENCES}

[1] D. G. Luenberger, "Observing the state of a linear system," IEEE transactions on military electronics, vol. 8, no. 2, pp. 74-80, 1964.

[2] D. Luenberger, "Observers for multivariable systems," IEEE Transactions on Automatic Control, vol. 11, no. 2, pp. 190-197, 1966.

[3] J. Doyle, "Guaranteed margins for lqg regulators," IEEE Transactions on Automatic Control, vol. 23, no. 4, pp. 756-757, 1978.

[4] J. Doyle and G. Stein, "Robustness with observers," IEEE Transactions on Automatic Control, vol. 24, no. 4, pp. 607-611, Aug 1979.

[5] J. P. Gauthier, H. Hammouri, and S. Othman, "A simple observer for nonlinear systems applications to bioreactors," IEEE Transactions on automatic control, vol. 37, no. 6, pp. 875-880, 1992. 

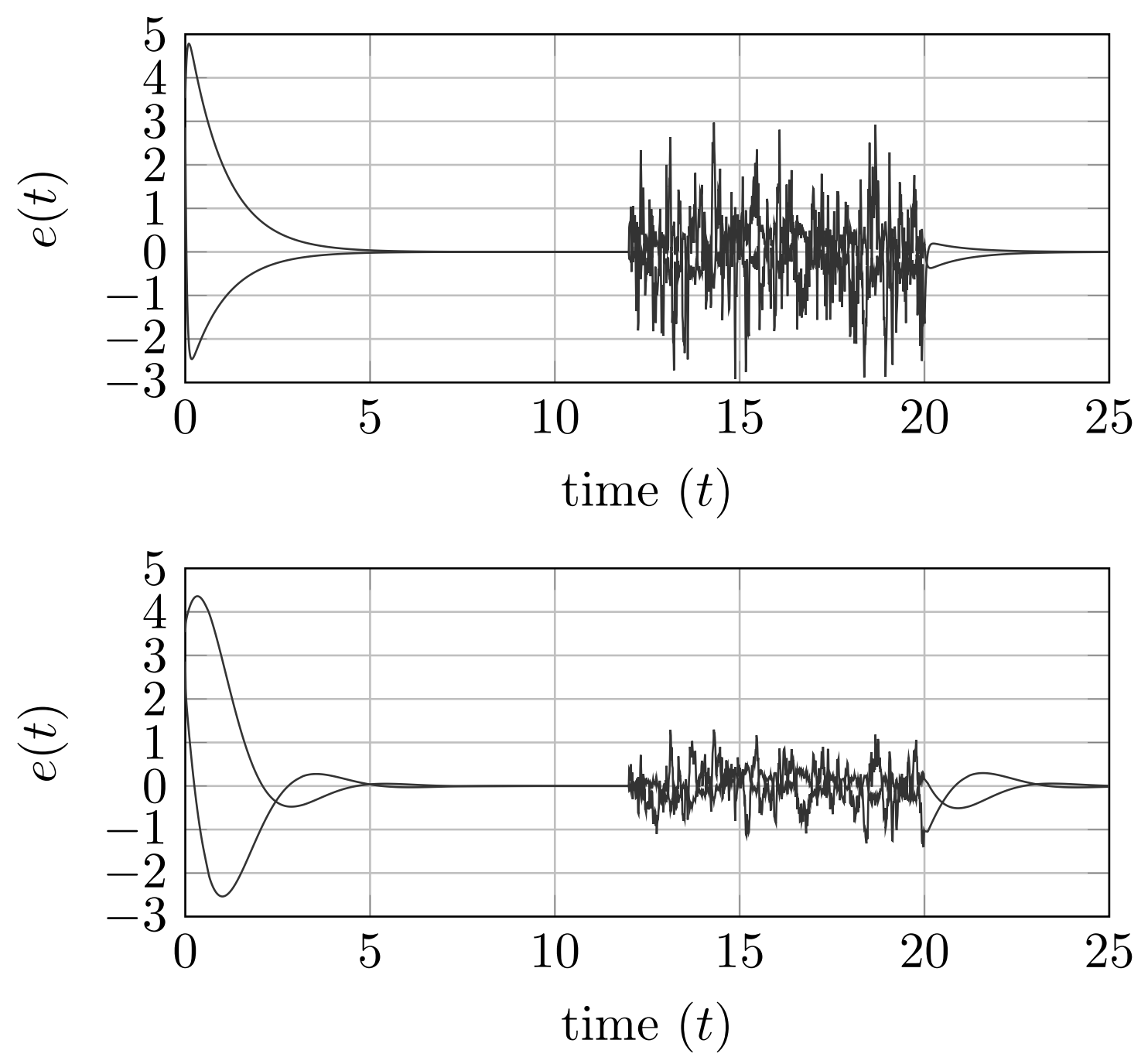

Fig. 2. Estimation error for the Luenberger observer (above) and the dead-zone observer (below). Notice that the dead-zone mechanism slightly deteriorates the transient performance.

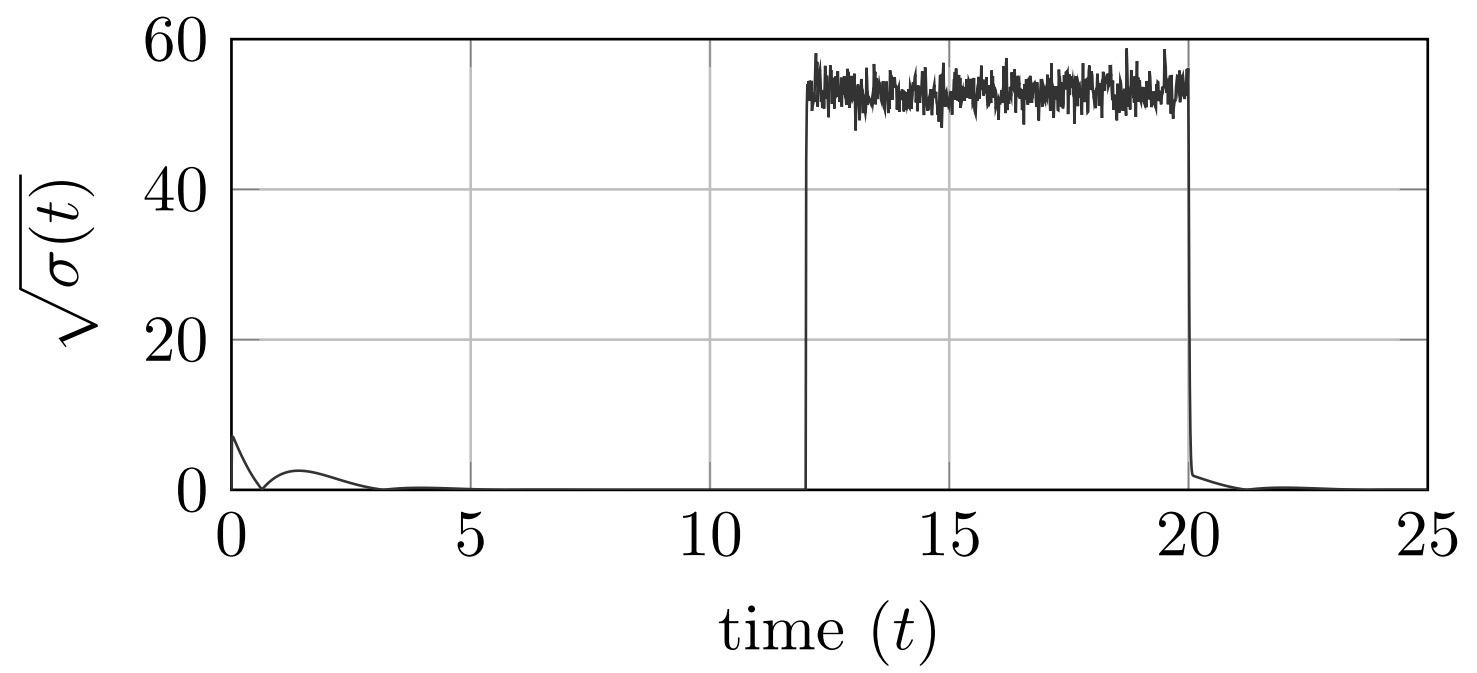

Fig. 3. Adaptation of the $\sigma$ level according to the noise level $v$. Notice that the large value of $\Lambda$ produces an almost immediate response in presence of noise. 
[6] F. Esfandiari and H. K. Khalil, "Output feedback stabilization of fully linearizable systems," International Journal of control, vol. 56, no. 5, pp. 1007-1037, 1992.

[7] A. Teel and L. Praly, "Tools for semiglobal stabilization by partial state and output feedback," SIAM Journal on Control and Optimization, vol. 33, no. 5 , pp. 1443-1488, 1995.

[8] - "Global stabilizability and observability imply semi-global stabilizability by output feedback," Systems \& Control Letters, vol. 22, no. 5, pp. 313-325, 1994.

[9] A. N. Atassi and H. K. Khalil, "A separation principle for the stabilization of a class of nonlinear systems," IEEE Transactions on Automatic Control, vol. 44, no. 9, pp. 1672-1687, 1999.

[10] H. Kwakernaak and R. Sivan, Linear optimal control systems. Wiley-Interscience New York, 1972, vol. 1.

[11] L. K. Vasiljevic and H. K. Khalil, "Error bounds in differentiation of noisy signals by high-gain observers," Systems \& Control Letters, vol. 57, no. 10, pp. 856-862, 2008.

[12] A. A. Prasov and H. K. Khalil, "Tracking performance of a high-gain observer in the presence of measurement noise," International Journal of Adaptive Control and Signal Processing, vol. 30, no. 8-10, pp. 1228-1243, 2016.

[13] D. Mayne, R. Grainger, and G. Goodwin, "Nonlinear filters for linear signal models," IEE Proceedings-Control Theory and Applications, vol. 144, no. 4, pp. 281-286, 1997.

[14] M. S. Chong, D. Nešić, R. Postoyan, and L. Kuhlmann, "Parameter and state estimation of nonlinear systems using a multi-observer under the supervisory framework," IEEE Transactions on Automatic Control, vol. 60, no. 9, pp. 2336-2349, 2015.

[15] J. H. Ahrens and H. K. Khalil, "High-gain observers in the presence of measurement noise: A switched-gain approach," Automatica, vol. 45, no. 4, pp. 936-943, 2009.

[16] A. A. Prasov and H. K. Khalil, "A nonlinear high-gain observer for systems with measurement noise in a feedback control framework," IEEE Transactions on Automatic Control, vol. 58, no. 3, pp. 569-580, 2013.

[17] R. G. Sanfelice and L. Praly, "On the performance of high-gain observers with gain adaptation under measurement noise," Automatica, vol. 47, no. 10, pp. 2165-2176, 2011.

[18] A. Alessandri and A. Rossi, "Increasing-gain observers for nonlinear systems: Stability and design," Automatica, vol. 57, pp. 180 - $188,2015$.

[19] V. Andrieu, L. Praly, and A. Astolfi, "Homogeneous approximation, recursive observer design, and output feedback," SIAM Journal on Control and Optimization, vol. 47, no. 4, pp. 1814-1850, 2008.

[20] _ _ "High gain observers with updated gain and homogeneous correction terms," Automatica, vol. 45, no. 2, pp. 422-428, 2009.

[21] H. K. Khalil, Nonlinear systems, 2nd ed. Upper Saddle River, (N.J.): Prentice Hall, 1996.

[22] J. G. Da Silva and S. Tarbouriech, "Antiwindup design with guaranteed regions of stability: an lmi-based approach," IEEE Transactions on Automatic Control, vol. 50, no. 1, pp. 106-111, 2005.

[23] T. Hu, Z. Lin, and B. M. Chen, "An analysis and design method for linear systems subject to actuator saturation and disturbance," Automatica, vol. 38, no. 2, pp. 351-359, 2002.

[24] T. Hu, A. R. Teel, and L. Zaccarian, "Anti-windup synthesis for linear control systems with input saturation: Achieving regional, nonlinear performance," Automatica, vol. 44, no. 2, pp. $512-519,2008$.

[25] S. Boyd, L. El Ghaoui, E. Feron, and V. Balakrishnan, "Linear matrix inequalities in system and control theory," 1994.

[26] A. Tilli and M. Montanari, "A low-noise estimator of angular speed and acceleration from shaft encoder measurement," Automatika-Zagreb, vol. 42, no. $3 / 4$, pp. $169-176,2001$.

[27] A. Alessandri and L. Zaccarian, "Results on stubborn luenberger observers for linear time-invariant plants," in 2015 European Control Conference (ECC), July 2015, pp. 2920-2925.

[28] E. D. Sontag, "An algebraic approach to bounded controllability of linear systems," International Journal of Control, vol. 39, no. 1, pp. 181-188, 1984.

[29] J. P. Hespanha, Linear systems theory. Princeton university press, 2009.

[30] H. Shim, J. Seo, and A. Teel, "Nonlinear observer design via passivation of error dynamics," Automatica, vol. 39, no. 5, pp. 885 - $892,2003$.

[31] M. Chilali and P. Gahinet, "H infinity; design with pole placement constraints: an lmi approach," IEEE Transactions on Automatic Control, vol. 41, no. 3, pp. 358-367, Mar 1996. 